\title{
HIGH RESOLUTION AIRBORNE SAR IMAGE CHANGE DETECTION IN URBAN AREAS WITH SLIGHTLY DIFFERENT ACQUISITION GEOMETRIES
}

\author{
E. Mendez Dominguez, D. Henke, D. Small, E. Meier \\ Remote Sensing Laboratories RSL, Department of Geography, University of Zurich, CH-8057 Zurich, Switzerland \\ (elias.mendez, daniel.henke, david.small, erich.meier)@geo.uzh.ch
}

KEY WORDS: Change Detection, SAR, Remote Sensing, Imaging Geometry, Feature, Building

\begin{abstract}
:
Before applying change detection, high resolution SAR (Synthetic Aperture Radar) imagery benefits from advanced denoising mechanisms to preserve details and minimize speckle. We propose a change detector based on a MCA (Morphological Components Analysis) of a difference image (DI). With MCA, the data is decomposed into image features utilizing sparse representations of the image content. By introducing a priori knowledge of the content of the scenes, and exploiting shape information corresponding to the changes provided by MCA, we can significantly improve performance under adverse conditions, such as inconsistent acquisition geometries.
\end{abstract}

\section{INTRODUCTION}

Image change detection is an enhancement technique that compares two or more images of the same area acquired at different times. The procedure interrogates a test image with respect to a reference image to obtain a binary map describing the changes. This method is particularly useful for Earth environmental observation, risk management, agricultural surveys, and urban studies. SAR sensors are well suited as time series data can be reliably collected; moreover, these sensors can operate in unfavorable weather and daylight conditions. Previous studies (Brunner, 2010; Bovolo, 2007; Schmitt, 2009) showed great potential for SAR-based image change detection. Nonetheless, the inherent complexity of SAR data imposes multiple challenges that need to be tackled.

Most of both coherent and incoherent SAR-based change detectors are designed to mitigate the errors due to the presence of noise. In image change detection, denoising carries an implicit trade-off between detail preservation and accurate representation of homogeneous areas. Some incoherent change detectors were proposed to assess this compromise and evaluated with high resolution spaceborne SAR imagery (Bovolo, 2007). A similar approach was presented with medium resolution data sets in (Celik, 2010a). All these methods do not filter, but instead exploit a multiresolution sequence, in which the noise level decreases with the resolution scale. A more typical example with explicit denoising can be found in (Celik, 2010b) and (Yousif, 2013).

Another alternative based on a wavelet fusion kernel that avoids direct denoising of the original SAR images was presented in (Ma, 2012). The low and high frequency components of the mean ratio and the log ratio images are fused with a decimated wavelet transform. Other methods based on the modification of the coefficients obtained in a transformed domain of the original image space were introduced in (Schmitt, 2009). In those, different enhancement techniques were applied to the curvelet coefficients of the difference image to highlight the structure of man-made objects.

Coherent change detectors are a good alternative to reduce the effect of the speckle noise without explicit suppressing noise in the SAR data (Damini, 2013; Wright, 2005; Scheuchl, 2009). Nonetheless, these detectors require short-term scenarios and data sets recorded with very similar flight paths, so that coherence is still a useful metric to evaluate changes. Different methods to perform coherent change detection were also proposed in (Scheuchl, 2009) and (Ridd, 1998), where the phase of the complex values of the SAR images is applied to a variant of Principal Components Analysis (PCA) rather than the coherence. PCA is highly sensitive to inter-channel correlation, thus the performance of this algorithm depends on the radiometry of the SAR images. The use of a sliding window can effectively remove this dependency at the cost of border artifacts. An efficient solution that mitigates these border effects was proposed in (Ranney, 2006).

To overcome some of the limitations of current coherent and incoherent SAR-based change detectors, particularly as applied to high resolution airborne SAR imagery, we propose advanced MCA-based denoising techniques to (a) accurately preserve details and suppress speckle and (b) cope with slightly inconsistent acquisition geometries (common for airborne SAR). For (b), we use the shape information inherited from MCA to remove some features caused by linear urban structures to improve the performance of the change detector.

This paper is organized into three sections. Section 2 introduces the design of the change detector when considering noise as the main source of error, and the required modifications to handle some errors induced by the different acquisition geometries for single channel airborne SAR data sets. Section 3 illustrates the results obtained with real multitemporal SAR data, showing the performance of the algorithm in typical urban scenarios. Finally, section 4 wraps up the document with a discussion of the results and conclusions.

\section{METHOD}

\subsection{Data and Test Sites}

The data sets were acquired with the DLR F-SAR sensor (Reigber, 2013) over the residential and industrial area of Oensingen (Switzerland) from three parallel paths, referred to as P0302, P0305 and P0306. The SAR images were recorded at X- 
band in fully polarimetric mode in linear stripmap configuration. To reflect typical variations in airborne acquisition geometries, Table 1 includes the incidence angle at mid-range of the different flight passes. Their geometries are illustrated in Figure 1. All SAR images shown in this document are single-look representations of the $\mathrm{HH}$ co-polarized channel after absolute radiometric and polarimetric calibration, with a theoretical range and azimuth resolution of 0.51 and 0.15 meters respectively. Absolute geometric calibration was achieved by using the geolocation of the corner reflectors deployed along the area of interest, giving residual range and azimuth geolocation errors below 2 centimetres in each dimension. The RAW data was processed with a matched filter without taper in range, and an interpolated matched filter (Graphics Processor Unit-based back-projection) with a Hanning tapper in azimuth (Henke, 2015).

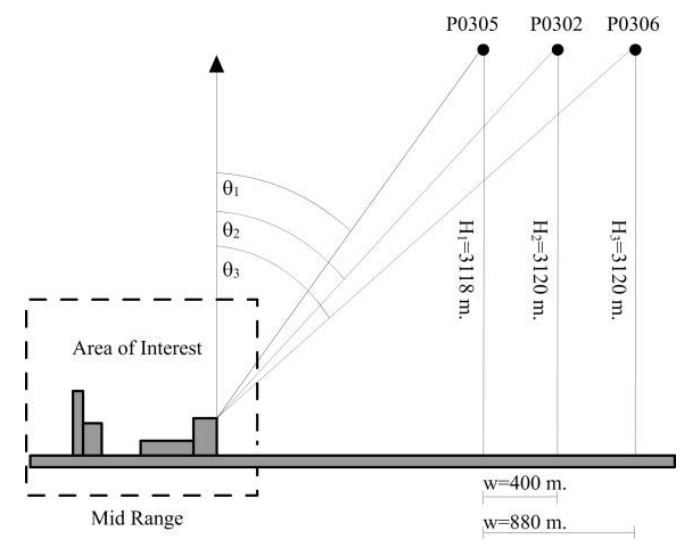

Figure 1. Geometry acquisition differences between the passes P0302, P0305 and P0306.

\begin{tabular}{|c|c|c|}
\hline $\begin{array}{c}\text { Flight } \\
\text { Pass }\end{array}$ & $\begin{array}{c}\text { Acquisition Date and } \\
\text { Time }\end{array}$ & $\begin{array}{c}\text { Incidence Angle at Mid- } \\
\text { Range }\left(\theta_{\mathrm{i}}\right)\end{array}$ \\
\hline P0302 & $16 / 06 / 201009: 23: 00$ & $47^{\circ}$ \\
\hline P0305 & $16 / 06 / 201009: 55: 15$ & $42.66^{\circ}$ \\
\hline P0306 & $16 / 06 / 201010: 06: 16$ & $51.34^{\circ}$ \\
\hline
\end{tabular}

Table 1. Acquisition characteristics.

\subsection{Morphological Components Analysis with SAR images}

Coherent interference of the electromagnetic waves reflected by the objects causes pixel-to-pixel intensity variations, manifesting as a granular noise pattern known as speckle (Lee, 2009). In incoherent SAR change detection, speckle affects the visual interpretation of the images, and can cause high false alarm rates and mis-detections. To mitigate these unwanted effects, noise treatment techniques are required. Let us assume that a log-transformed amplitude SAR image is expressed as:

$$
\mathrm{x}=\mathrm{y}+\varepsilon
$$

where $\mathrm{x}$ is the noise-corrupted log-amplitude SAR image, $\mathrm{y}$ is the image to recover, and $\varepsilon$ is additive noise whose properties are unknown a priori.

In this work we focus on noise suppression methods that work in a transformed domain with multiresolution decomposition and shrinking rules. The denoised image is recovered after back transforming the modified coefficients obtained in the forward transform. The design of these methods is based on the compressed sensing theorem (Starck, 2010), where it is assumed that the transform is able to represent the data in a sparse form. The preservation of details and appearance of artifacts are therefore linked to the veracity of this assumption, together with the accuracy of the shrinking rules and the thresholds involved. For instance, wavelets are known to provide a sparse representation for smooth or uniform areas and point-like targets, but fail to represent other types of image content as lines or edges. Multiple transforms that can compactly represent different types of data are then good alternatives to alleviate over-smoothing and artifacts. For this reason, and based on the basic principle of morphological diversity it is desirable to account for the different geometric features found in high resolution SAR imagery, exploiting the advantages of different morphological transforms when used in combination (Starck, 2010). In this manner, a SAR image $x$ is expressed as the combination of $\mathrm{K}$ transforms $\Psi$, when $\mathrm{x}$ is corrupted with additive Gaussian noise $\varepsilon$ (with zero mean and standard deviation $\sigma_{\varepsilon}$ ):

$$
\mathrm{x}=\sum_{\mathrm{k}=1}^{\mathrm{K}} \Psi_{\mathrm{k}} \alpha_{\mathrm{k}}+\varepsilon
$$

where $\alpha_{\mathrm{k}}$ is the matrix of coefficients of $\mathrm{y}$ in the transformed domain $\Psi_{\mathrm{k}}$. An approximate solution to (2) can be found via MCA. This algorithm restricts the space of possible solutions or candidates by modelling a constraint optimization problem as follows:

$$
\min _{\alpha_{1}, \ldots, \alpha_{\mathrm{K}}} \sum_{\mathrm{k}=1}^{\mathrm{K}}\left\|\alpha_{\mathrm{k}}\right\|_{1}^{1} \text { s.t. }\left\|\mathrm{x}-\sum_{\mathrm{k}=1}^{\mathrm{K}} \Psi_{\mathrm{k}} \alpha_{\mathrm{k}}\right\|_{2} \leq \sigma
$$

We need to find the set of coefficients $\alpha_{\mathrm{k}}$ of the K transforms which have the minimum $\ell_{1}$ norm (which produces a more sparse representation) and provide the minimum reconstruction error $\sigma$ (fidelity to data). If all the components $\alpha_{1}$ but $\alpha_{k}$ are fixed, we can find a solution by soft-thresholding the coefficients of the marginal residuals $\mathrm{r}_{\mathrm{k}}$, computed as:

$$
\mathrm{r}_{\mathrm{k}}=\mathrm{x}-\sum_{\mathrm{l} \neq \mathrm{k}} \Psi_{1} \alpha_{1}
$$

The soft-thresholding shrinking rule is defined as:

$$
\text { SoftThresh }(X, T)=\operatorname{sign}(X)(|X|-T)
$$

where $\mathrm{X}$ is a generic real variable and the threshold $\mathrm{T}$ is computed via:

$$
\mathrm{T}=3 * \operatorname{median}\left(\left|\mathrm{w}_{3}-\operatorname{median}\left(\mathrm{w}_{3}\right)\right|\right) / \mathrm{c}_{0}
$$

with $w_{3}$ being the coefficients of the diagonal subband of the Haar wavelet of the input image $\mathrm{x}$ obtained at the first resolution scale, and $\mathrm{c}_{0}=0.6745$ (Starck, 2010; Mallat, 2008).

The evaluation of (4) is the basis of the iterative kernel of the MCA algorithm used here. A break evaluates the performance of the method at each iteration. We opted for adaptive MCAMOM (Mean of Maximum) because it provides better performance when the components have high contrast, assuring that each transform gets at least one assigned component (Starck, 2010). Nonetheless, and due to mathematical limitations, all targets are expressed as a combination of all the transforms involved, and thus it is not possible to guarantee a perfect separation of shapes. Finally, after reaching the stopping 
conditions, the output is defined as the morphological components $\mathrm{x}_{\mathrm{k}=1 \ldots \mathrm{K}}^{\mathrm{Niter}}$ of the input image $\mathrm{x}$. In this paper we exploit only the $\mathrm{K}$ components of the last iteration.

\subsection{MCA-based Change Detection}

A SAR change detector involves two main stages. First, a mathematical function $f$ is applied to the SAR images $\mathrm{x}_{1}$ and $\mathrm{x}_{2}$ recorded at two different time periods over the same geographical area. In this work we exploit a so-called Difference Image (DI), obtained when $f$ is the difference between log-transformed $x_{1}$ and $x_{2}$. The subtraction is still the most common operator in SAR change detection, although other alternatives exist (Damini, 2013; Yousif, 2013; Ridd, 1998). A binary map is obtained by thresholding the DI. This map contains the detected changes in the form of classes (change or no change).

To suppress most of the noise-induced errors inherently present in the DI, the data is decomposed into different image features using sparse representations of the image content by deploying a wavelet- and curvelet-based MCA, $\mathrm{K}=2$ in equations (2-4). To avoid aliasing effects and guarantee translation invariance, we use a stationary wavelet transform (SWT) designed with the classic 'a troux' method. This wavelet transform provides better image restoration properties than decimated transforms (Starck, 2010). However, Gibbs-like artifacts, or ringing, produced by this harmonic analysis introduces multiple false alarms, and thus, an additional strategy is required to remove them from the binary map. Due to translation invariance of the stationary wavelet transform, a cycle spinning method (Starck, 2010) does not yield improved results. We utilize a second resolution scale of the DI, in a fashion similar to decimated pyramid transforms (Starck, 2010). This additional scale is down-sampled by a factor $\mathrm{L}$ using an interpolation kernel that maintains the noise properties for the whole chain, such as Lanczos (Burger, 2012). The resulting resolution scale is referred as the $\mathrm{DI}_{\mathrm{d}}$. The two resolution scales are decomposed by MCA independently, with the curvelet transform remaining unchanged and the wavelet transform using a different wavelet base for each scale. For the second scale the mother wavelet has more vanishing moments, as the down-sampled DI contains less speckle (Mallat, 2008). After applying MCA to each resolution scale, the DI and the $\mathrm{DI}_{\mathrm{d}}$ are re-expressed as:

$$
\begin{gathered}
\mathrm{DI}_{\mathrm{MCA}}=\Psi_{\mathrm{CURV}} \alpha_{\mathrm{CURV}}+\Psi_{\mathrm{SWT}-\mathrm{db} 4} \alpha_{\mathrm{SWT}-\mathrm{db} 4} \\
\mathrm{DI}_{\mathrm{d}-\mathrm{MCA}}=\Psi_{\mathrm{CURV}} \alpha_{\mathrm{CURV}}^{\mathrm{d}}+\Psi_{\text {SWT-db8 } 8} \alpha_{\text {SWT-db8 }}^{\mathrm{d}}
\end{gathered}
$$

where $\alpha_{\text {CURV }}$ and $\alpha_{\text {CURV }}^{\mathrm{d}}$ are the denoised curvelet coefficients (i.e. iteratively soft-thresholded with Eq. (5)) of the DI and $\mathrm{DI}_{d}$ respectively. The sets $\alpha_{\text {SWT-db4 }}$ and $\alpha_{\text {SWT-db8 }}^{\mathrm{d}}$ are the denoised wavelet coefficients of the DI and the $\mathrm{DI}_{\mathrm{d}}$ extracted with an Ingrid-Daubechies mother wavelet (Mallat, 2008) with 4 and 8 vanishing moments respectively.

Note that the exploitation of two resolution scales introduces multiple benefits; for instance, it is very effective at mitigating ringing or producing a migration of the artifacts to different image pixels; downsampling is the simplest noise suppresion mechanism to be integrated in a change detector; and noise supression of a downsampled image is better than suppressing the noise in an image and downsampling it (Burger, 2012).

The global binary map is finally obtained with:

$$
\mathrm{F}=\mathrm{F}_{1} \wedge \mathrm{F}_{2}=\left(\left|\mathrm{DI}_{\mathrm{MCA}}\right| \leq \mathrm{T}_{1}\right) \wedge\left(\left|\left(\mathrm{DI}_{\mathrm{d}-\mathrm{MCA}}\right) \uparrow \mathrm{L}\right| \leq \mathrm{T}_{1}\right)
$$

where $\uparrow \mathrm{L}$ represents an upsampling operator by a factor $\mathrm{L}$, and $F_{1}$ and $F_{2}$ are the binary maps of each resolution scale after thresholding with $\mathrm{T}_{1}$.

Additionally, with the shape information of the detected changes obtained via MCA, the change detector can account for some errors induced by differences between acquisition geometries. If no additional data in the form of digital surface models, interferometric or tomographic SAR imagery is available, some a priori knowledge of the content of the scene is therefore required; for instance in urban areas, the PSFs (Point Spread Functions) of the walls/roofs of the buildings migrate to different pixels among the data takes. We exploit the shape information obtained with MCA to suppress such geometry-based changes (i.e. lines, edges, contours...) while preserving real changes on the ground. In this manner, to remove the changes detected with a particular morphological component, we first threshold and combine the two binary maps of that component with:

$$
\mathrm{F}_{\text {curv }}=\left(\left|\Psi_{\mathrm{CURV}} \alpha_{\mathrm{CURV}}\right| \leq \mathrm{T}_{1}\right) \wedge\left(\left|\left(\Psi_{\mathrm{CURV}} \alpha_{\mathrm{CURV}}^{\mathrm{d}}\right) \uparrow \mathrm{L}\right| \leq \mathrm{T}_{1}\right)
$$

Finally we extract those changes from the global binary map using:

$$
\mathrm{G}=\mathrm{F} \wedge \mathrm{F}_{\text {curv }}
$$

In this work, we use $T_{1}=3$ decibels, chosen as the typical metric used to evaluate the resolution of a SAR image, in order to guarantee that when the point-like targets enter or leave the scene the changes are resolved in both range and azimuth. Note that all binary maps are obtained with the same threshold $T_{1}$, although better results could be achieved if a particular component were evaluated using another set of thresholds.

\section{RESULTS}

The results are presented as an overlay of the binary map on top of the reference image. The maps contain two classes: the pixels marked in green/red indicate a significant increase/decrease of the radar brightness between the reference and test images, i.e. targets entered/left the area of interest. To ease the interpretation of the different targets, we include an aerial view of the areas of interest (Figure 2 and Figure 3). Corresponding RGB composites are shown in Figure 4 (a-b) and Figure 5 (a-b) respectively, produced with the reference image in the red channel and the test image in the green channel. In this manner, the most remarkable changes are highlighted in red and green, whereas yellow indicates a low probability of change.

Figure 4 illustrates the changes detected in the industrial area of Oensingen. The right/left column of the figure contains the results when the acquisition geometries induce an incidence angle difference of 4 degrees/ 8 degrees respectively. Subfigures (c) and (d) show the output of the detector considering only noise-induced errors, whereas subfigures (e) and (f) are obtained after exploiting a priori knowledge of the acquisition geometries-induced changes. The same scheme was followed in Figure 5, where changes were detected from analysis of the residential area of the town.

The results reveal that the changes detected with the curvelet component via MCA can be related to walls/roofs of the buildings and are thus, isolated. In this manner, the false alarms induced by the migration of the PSFs of the linear shapes can be 
removed. Notice that due to the short-term scenario (see Table 1) only cars and trucks represent real changes on the ground; thus the rest of the detected changes must be considered as false alarms.

After manual inspection, subfigures 4 (e-f) contain most of the real changes, with the exception of some false alarms on the rooftops, which cannot be removed due to their blob-like shapes suited by the wavelet component. Subfigures 5 (e-f) have a more difficult visual evaluation; nonetheless, the changes detected in the parking area indicated by the blue rectangle reveal the movement of some cars, with a high likelihood of real change.

\section{DISCUSSION}

\subsection{Analysis of the Results}

Our algorithm was evaluated concentrating on two different areas of interest. The first was strongly dominated by long linear structures as is typical for buildings in industrial areas, whereas the second contains smaller linear contours more representative of low-medium residential areas.

When considering noise as the only source of error, we observed that the uniform areas of the image shown did not present false alarms. Due to the MCA inherited shapeproperties, well-defined structures such as edges, contours, cars, railways, and rooftops were clearly identifiable, generally outperforming the outputs provided by classical noise suppression methods.

When the acquisition geometries exhibit incidence angle differences lower than 4 degrees, we observed that the targets on the ground, such as cars and trucks, can be detected and retained after removing the curvelet component. For an incidence angle difference of 8 degrees, we observed that the migration of the PSFs was considerably higher, and thus some false alarms were still present in the binary map after the removal of the curvelet component. This issue is also likely to be more severe with increasing differences in the incidence angles of the acquisition geometries.

Utilizing the curvelet component, false alarms given by the artifacts produced by the processing chain, such as range/azimuth side-lobes and clutter areas, might be removed.

\subsection{Conclusions}

A novel change detector based on MCA was proposed to handle high resolution SAR imagery with a noise treatment able to preserve relevant details with well-defined structures. The method's detection performance expedited manual inspection of both the changes and their origin.

By exploiting the shape information, we can significantly improve the performance of the change detector when different acquisition geometries exist, given some prior knowledge about structures in the scene (as e.g. in urban areas). The method does not utilize additional SAR data, and it can be extended to retain other shapes by introducing their respective morphological components. However, for very different acquisition geometries additional data, such as multichannel SAR data sets or accurate digital surface models might be required. In future work we consider a detailed evaluation of the algorithm to circular geometries, as well as exploitation of the scattering mechanism classifications extracted from fully polarimetric data sets. Additionally, a more objective evaluation of the algorithm is currently on-going considering similar acquisition geometries, in areas where ground truth was recorded.

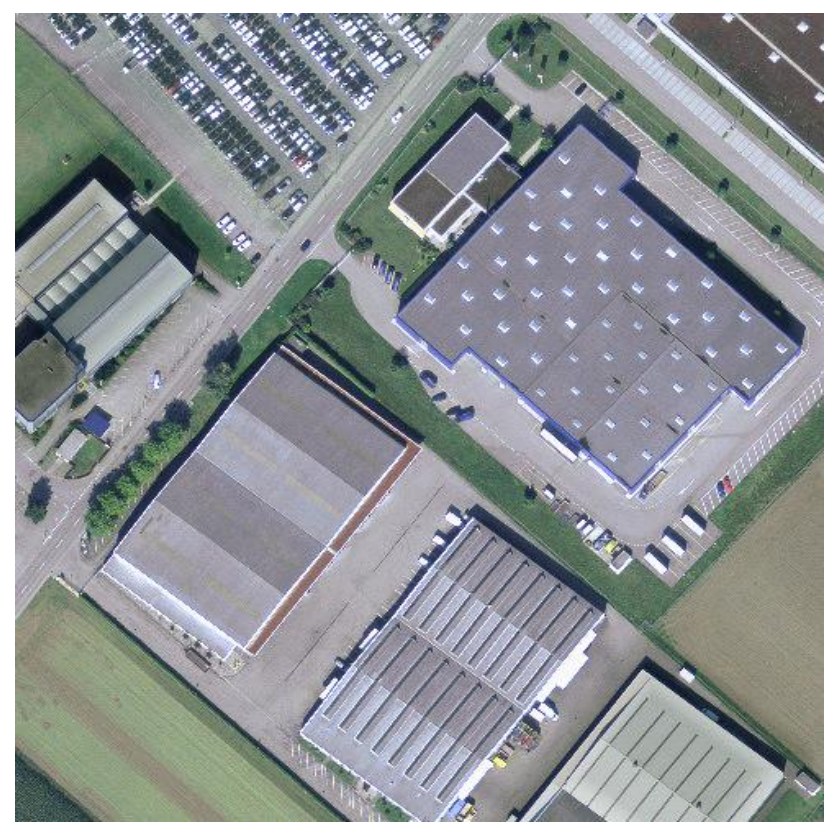

Figure 2. Orthophoto of the industrial neighbourhood of Oensingen, (@ swisstopo, 2006).

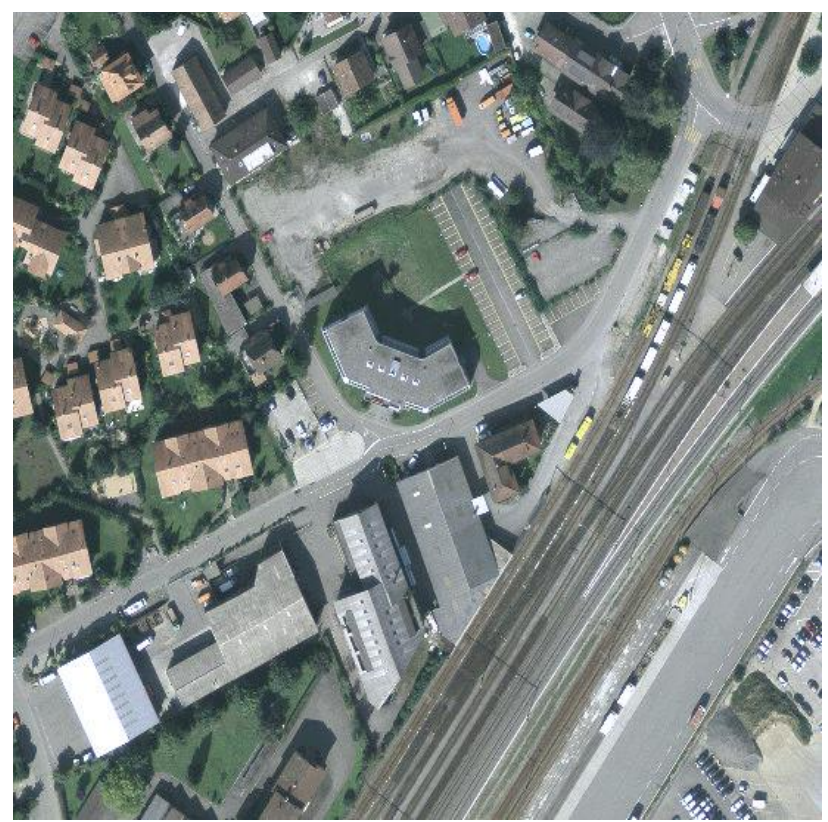

Figure 3. Orthophoto of the residential neighbourhood of Oensingen, (@ swisstopo, 2006). 


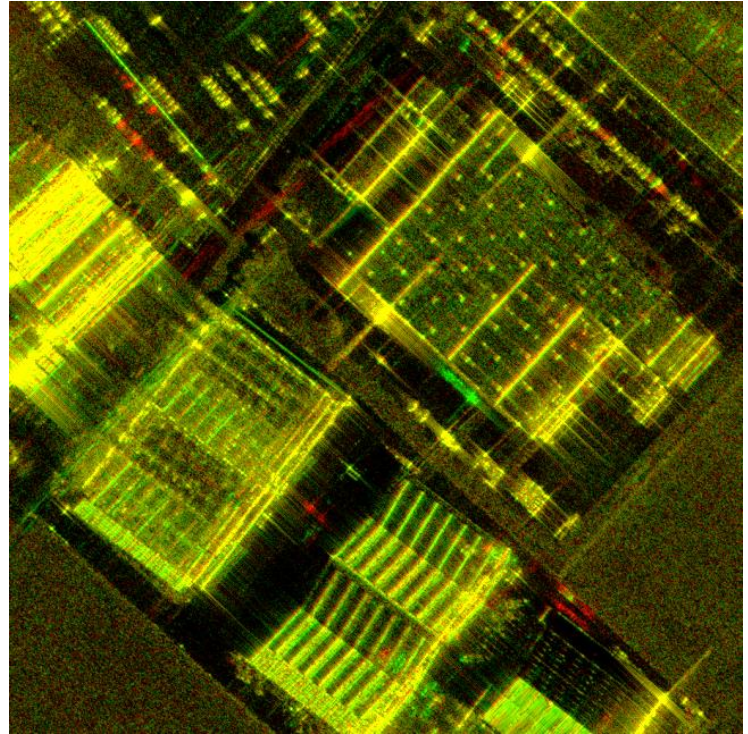

(a) RGB composite: $\mathrm{R}=\mathrm{P0302}, \mathrm{G}=\mathrm{P0305}$

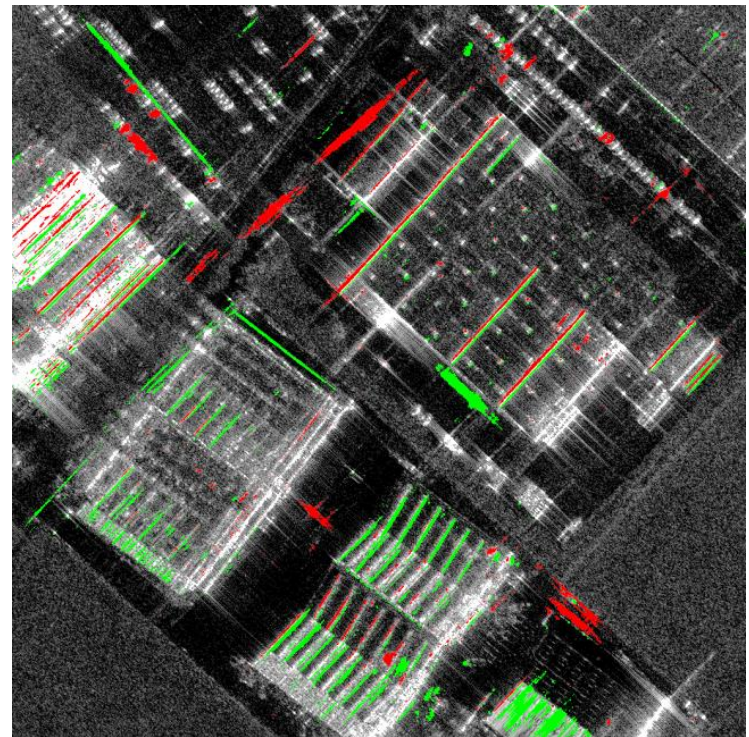

(c) CD-MCA (all components): P0302-P0305

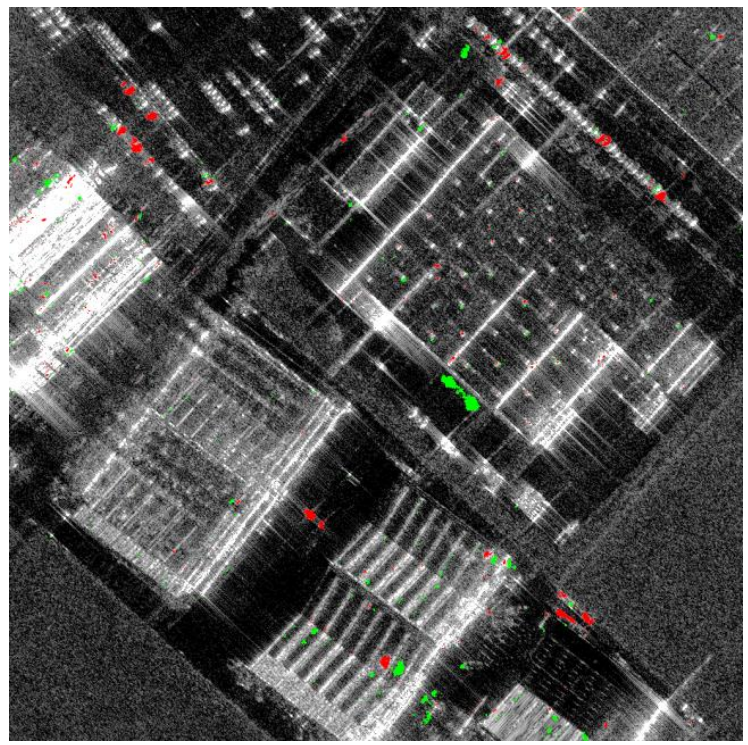

(e) CD-MCA (without curvelet component): P0302-P0305

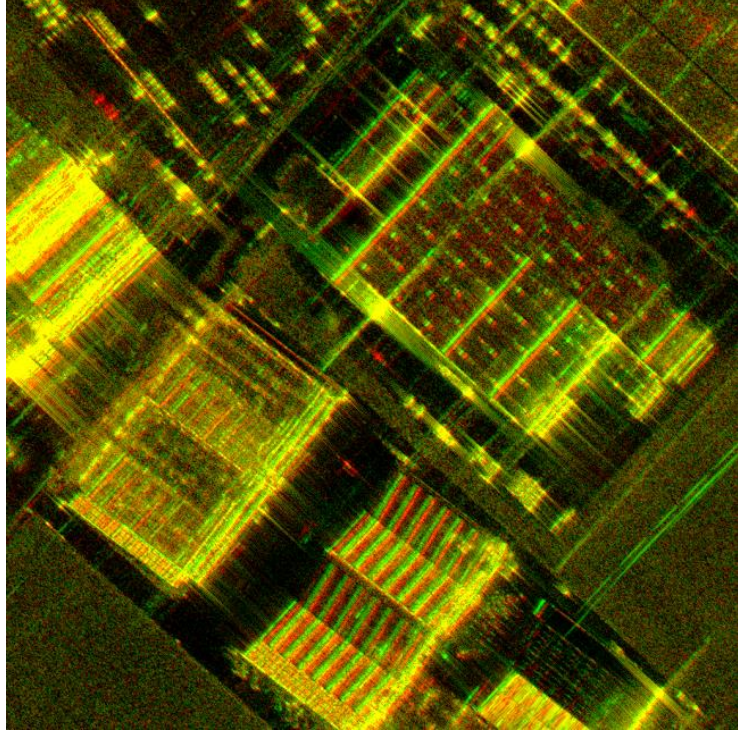

(b) RGB composite: $\mathrm{R}=\mathrm{P0305}, \mathrm{G}=\mathrm{P0306}$

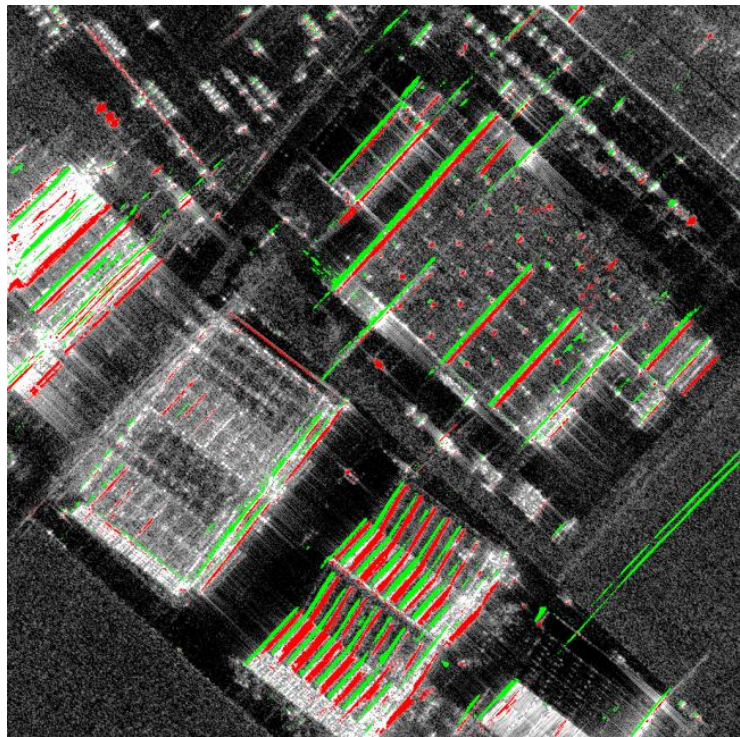

(d) CD-MCA (all components): P0305-P0306

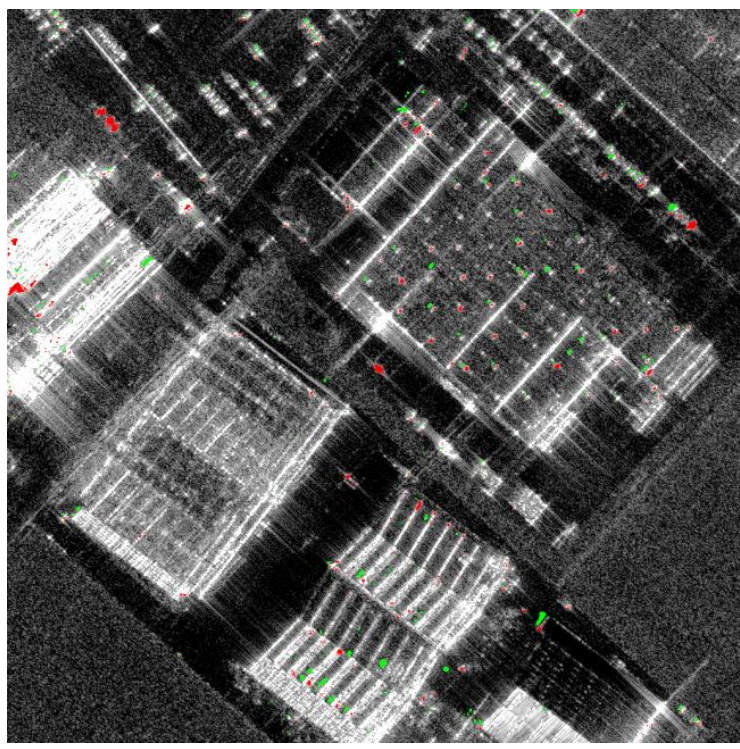

(f) CD-MCA (without curvelet component): P0305-P0306

Figure 4. Results of the change detector in the industrial neighbourhood. 


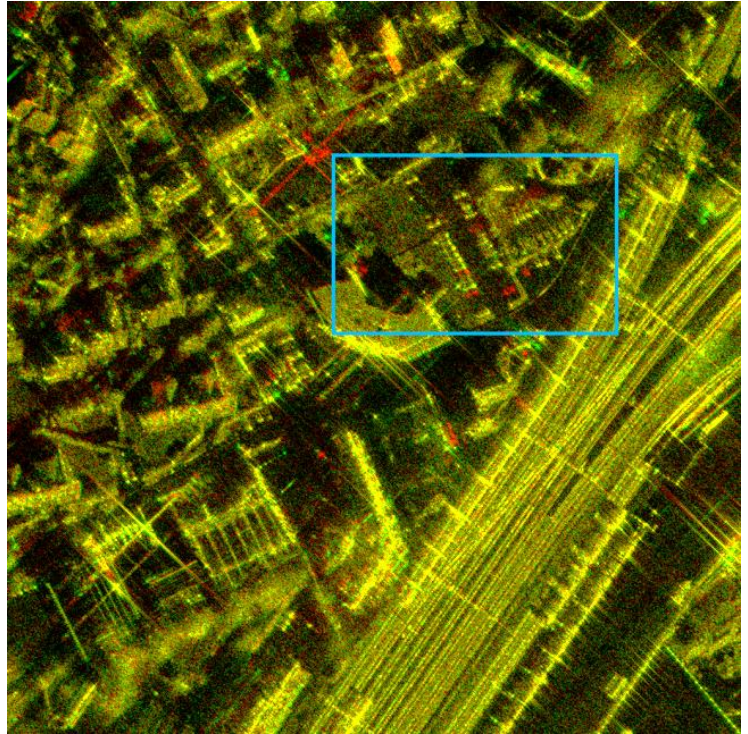

(a) RGB composite: $\mathrm{R}=\mathrm{P} 0302, \mathrm{G}=\mathrm{P0305}$

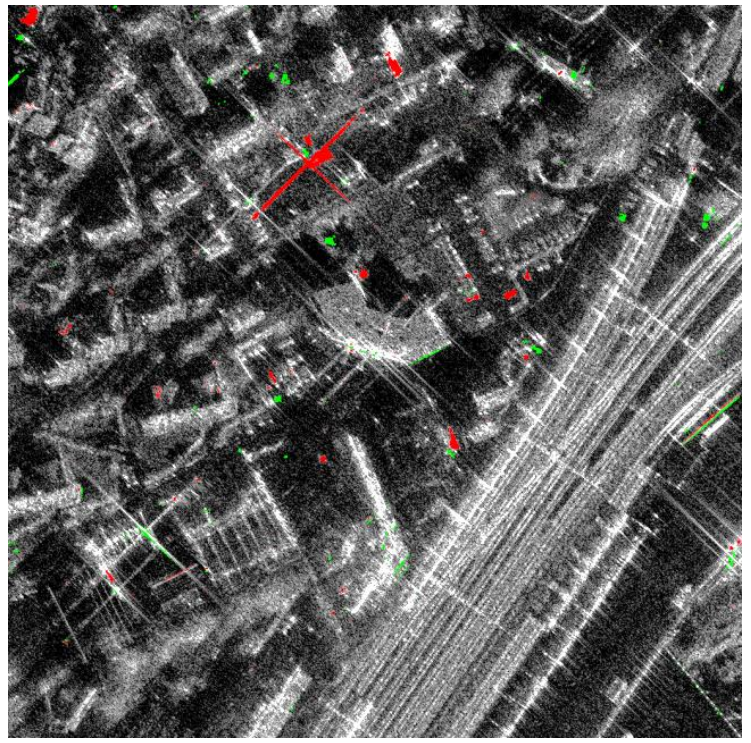

(c) CD-MCA (all components): P0302-P0305

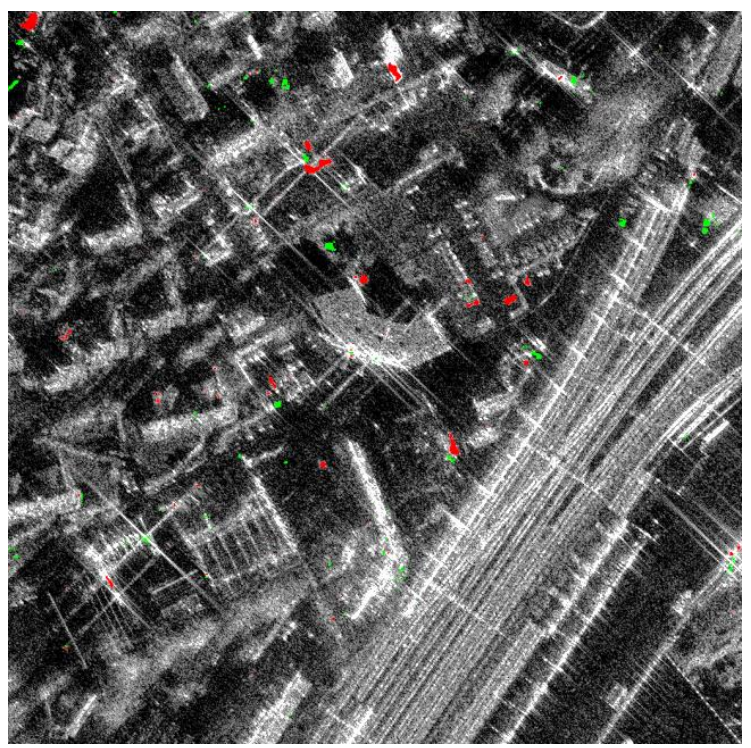

(e) CD-MCA (without curvelet component): P0302-P0305

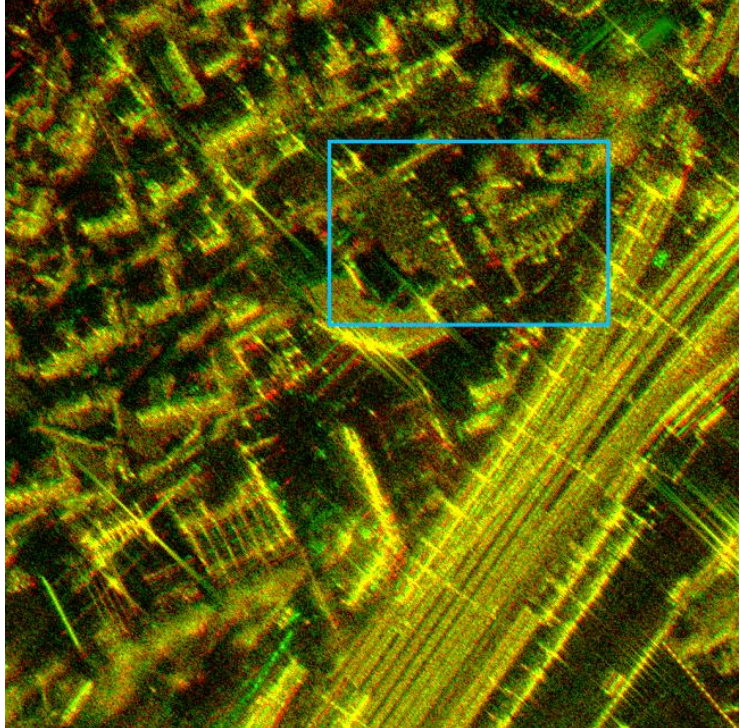

(b) RGB composite: $\mathrm{R}=\mathrm{P0305}$, G=P0306

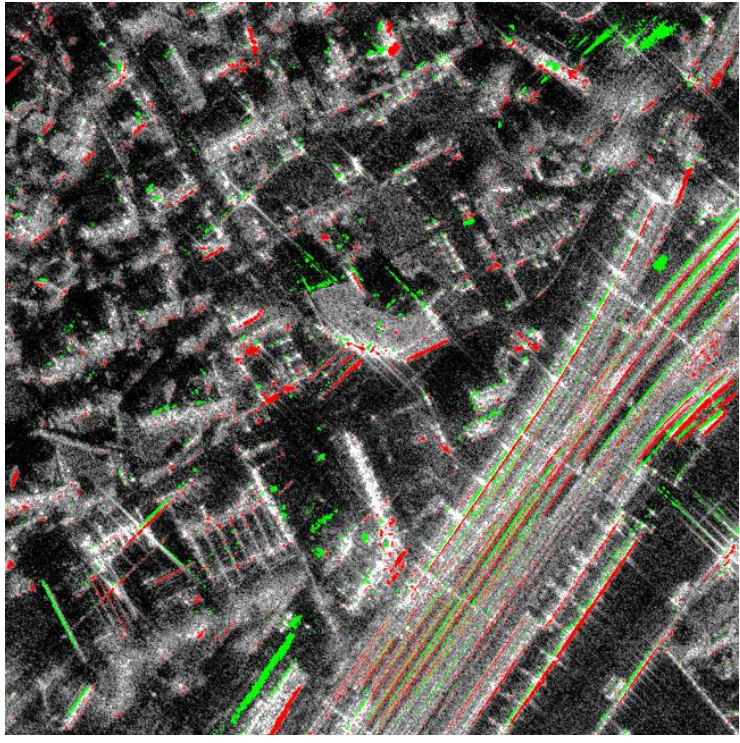

(d) CD-MCA (all components): P0305-P0306

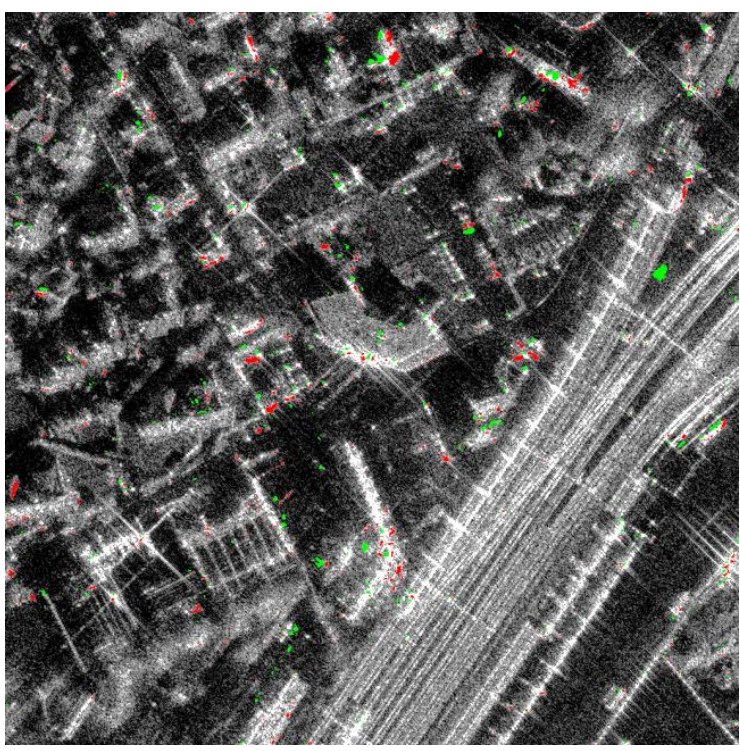

(f) CD-MCA (without curvelet component): P0305-P0306

Figure 5. Results of the change detector in the residential neighbourhood. 


\section{REFERENCES}

Bovolo F., and Bruzzone L., 2005. A Detail-Preserving ScaleDriven Approach to Unsupervised Change Detection in Multitemporal SAR Images. IEEE Transactions on Geoscience and Remote Sensing, 43(12), pp. 2963-2972.

Bovolo F., and Bruzzone L., 2007. A Split-Based Approach to Unsupervised Change Detection in Large-Size Multitemporal Images: Application to Tsunami-Damage Assessment. IEEE Transactions on Geoscience and Remote Sensing, 45(6), pp. 1658-1670.

Brunner D., Lemone G., and Bruzzone L., 2010. Earthquake damage assessment of buildings using VHR optical and SAR imagery. IEEE Transactions on Geoscience and Remote Sensing, 48(5), pp. 2403-2420.

Burger H. C., 2012. Modelling and Learning Approaches to Image Denoising. PhD dissertation, Eberhard Karls University Tübingen.

Celik T., and Curtis C. V., 2010a. Resolution selective change detection in satellite images. IEEE International Conference on Acoustics Speech and Signal Processing (ICASSP), pp. 970-973.

Celik T., and Kuang K., 2010b. Unsupervised change detection for satellite images using dual-tree complex wavelet transform. IEEE Transactions on Geoscience and Remote Sensing, 48(3), pp. 1199-1210.

Dabov K., Foi A., Katkovnik V., and Egiazaran K., 2007. Image denoising by sparse 3D transform-domain collaborative filtering. IEEE Transactions on Image Processing, 16(8), pp. 2080-2095.

Damini A., Mantle V., and Davidson G., 2013. A new approach to coherent change detection in VideoSAR imagery using stack averaged coherence. IEEE International Radar Conference, pp. $1-5$.

Henke D., Mendez Dominguez E., Small D., Schaepman E. M., and Meier E., 2015. Moving Target Tracking in Single and Multichannel SAR. IEEE Transactions on Geoscience and Remote Sensing 53(6), pp. 3146-3159.

Lee J. S., and Pottier E., 2009. Polarimetric radar imaging: from basics to applications. CRC press.

Ma J., Gong M., and Zhou Z., 2012. Wavelet Fusion on Ratio Images for Change Detection in SAR Images. IEEE Geoscience and Remote Sensing Letters, 9(6), pp. 1122-1126.

Mallat, S., 2008. A wavelet tour of signal processing: the sparse way. Elsevier.

Ranney K. I., and Soumekh M., 2006. Signal subspace change detection in averaged multilook SAR imagery. IEEE Transactions on Geoscience and Remote Sensing, 44(1), pp. 201-213.
Reigber, A., Scheiber, R., Jager, M., Prats-Iraola, P., Hajnsek, I., Jagdhuber, T., Papathanassiou, K.P., Nannini, M., Aguilera, E., Baumgartner, S., Horn, R., Nottensteiner, A., and Moreira, A., 2013. Very-High-Resolution Airborne Synthetic Aperture Radar Imaging: Signal Processing and Applications. Proceedings of the IEEE, 101(3), pp.759-783.

Ridd M. K., and Jiajun L., 1998. A comparison of four algorithms for change detection in an urban environment. Remote sensing of environment, 63(2), pp. 95-100.

Scheuchl B., Ullmann T., and Koudogbo F., 2009. Change detection using high resolution Terrasar-X data - preliminary results. The International Archives of the Photogrammetry, Remote Sensing and Spatial Information Sciences, 38, pp. 1-4.

Schmitt, A., Wessel B., and Roth A., 2009. Curvelet approach for SAR image denoising, structure enhancement, and change detection. In: The International Archives of the Photogrammetry, Remote Sensing and Spatial Information Sciences. 38(3/W4), pp. 151-156.

Starck J. L., 2010. Sparse Image and Signal Processing: Wavelets, Curvelets and Morphological Diversity. Cambridge University Press.

Wright P., Macklin T., Willis C., and Rye T., 2005. Coherent change detection with SAR. IEEE European Radar Conference, pp. 17-20.

Yousif O., and Ban Y., 2013. Improving Urban Change Detection From Multitemporal SAR Images Using PCA-NLM. IEEE Transactions on Geoscience and Remote Sensing, 51(4), pp. 2032-2041. 